\title{
Development and evaluation of solid dispersion of spironolactone using fusion method
}

\author{
Shamsuddin, Mohammad Fazil ${ }^{1}$, Shahid Husain Ansari' ${ }^{2}$ Javed Ali $^{1}$ \\ Department of Pharmacy, OPJS University, Rajasthan, ${ }^{1}$ Departments of Pharmaceutics and ${ }^{2}$ Pharmacognosy, Jamia Hamdard, New Delhi, India
}

\begin{abstract}
Introduction: Solubility and dissolution of a poor water-soluble drug are the two major barriers for formulation scientists in development of drug delivery. Many of the potent drugs do not show the therapeutic effects due to solubility issues but may show toxicity issues when used in high doses. Solid dispersion (SD) technology is an excellent tool for enhancing the solubility and dissolution and hence related bioavailability. Materials and Methods: SD of spironolactone (SPL) was developed using an inert carrier polyethylene glycol 4000 (PEG 4000) by the conventional fusion method and characterized for various characterization parameters. Results: Solubility of pure drug and SD of SPL in water was found to be $23.54 \pm 1.75 \mu \mathrm{g} / \mathrm{mL}$ and $61.73 \pm 1.26 \mu \mathrm{g} / \mathrm{mL}$, respectively. The maximum cumulative percentage release from pure drug, SPL marketed product (tablet), physical mixture, and SPL SD at 60 min was $27.25 \pm 1.83 \%, 35.64$ $\pm 3.65 \%, 47.72 \pm 2.45 \%$, and $74.24 \pm 3.25 \%$, respectively in $0.1 \mathrm{~N} \mathrm{HCl}$. Conclusions: SD of SPL was developed successfully. The solubility of SPL SD was found to be significantly increased $(P<0.05)$ as compared to SPL active pharmaceutical ingredient (API) and physical mixture of PEG 4000 and SPL. The current study indicated that SD of SPL was a better option for enhancing solubility of a poorly soluble therapeutic agent.
\end{abstract}

Key words: Polyethylene glycol 4000 (PEG 4000), poor water solubility, solid dispersion (SD), spironolactone (SPL)

\section{INTRODUCTION}

Various drugs are synthesized by scientists but most compounds undergoing development at the present time have solubility and dissolution problems, which are key problems in drug discovery and product development for oral dosage forms. Compounds having limited solubility in water, typically below $0.1 \mathrm{mg} / \mathrm{mL}$, present unusual challenges in drug discovery. This is especially true in many circumstances for which solubilization and dissolution limit drug absorption. ${ }^{[1]}$ Various approaches have been applied for improvement in apparent solubility and dissolution rate including formation of soluble salts of drugs, reduction of crystals size, conversion of drugs into prodrugs, use of amorphous forms, cosolvation and superdisintegrants,

\section{Address for correspondence:}

Dr. Javed Ali,

Department of Pharmaceutics, Faculty of Pharmacy,

Jamia Hamdard, Hamdard Nagar, New Delhi - 110 062, India.

E-mail: javedaali@yahoo.com

\begin{tabular}{|l|l|}
\hline \multicolumn{2}{|c|}{ Access this article online } \\
\hline Quick Response Code: & Website: \\
\hline & www.jpionline.org \\
\cline { 2 - 2 } & DOI: \\
\hline
\end{tabular}

impregnating liquid drugs or drug solution in porous powders, using surface active self-emulsifying systems, micronization, formation of inclusion complexes with cyclodextrin, formation of amorphous drug, and formation of solid dispersion (SD) with hydrophilic carriers. ${ }^{[1-5]}$ Although salt formation and particle size reduction are commonly used to increase dissolution rate and oral absorption, there are some practical limitations for these techniques. The salt formation is not feasible for neutral compounds and weak electrolytes. On the other hand, very fine powders of hydrophobic drugs are difficult to disperse in water due to poor wettability. The loading of liquid drugs in porous powder (called "powder solutions") encountered flow property and compressibility issues. ${ }^{[6]}$

SD is now firmly established as a platform technology for the formulation of poorly soluble drugs. Specifically, SD technology

This is an open access article distributed under the terms of the Creative Commons Attribution-NonCommercial-ShareAlike 3.0 License, which allows others to remix, tweak, and build upon the work non-commercially, as long as the author is credited and the new creations are licensed under the identical terms.

For reprints contact: reprints@medknow.com

How to cite this article: Shamsuddin, Fazil M, Ansari SH, Ali J. Development and evaluation of solid dispersion of spironolactone using fusion method. Int J Pharma Investig 2016;6:63-8. 
has been successfully applied to develop formulations with a high drug and/or containing drugs with a high tendency to crystallize. ${ }^{[7]} \mathrm{SD}$ is defined as dispersion of drug in an amorphous polymer matrix where the drug is preferably in the molecularly dispersed state. ${ }^{[8,9]}$ These systems were defined as the dispersion of one or more active ingredients in an inert matrix in the solid state prepared by melting (fusion), solvent or melting solvent method with the goal of enhancing oral bioavailability (BA). ${ }^{[10]}$ These dosage forms have been traditionally regarded as being synonymous with the systems whereby the in vitro release of the drug is enhanced compared to conventional dosage forms, with concomitant implication for in vivo release. ${ }^{[3]}$ This technique has been used for a wide variety of poorly soluble drugs such as nimesulide, ketoprofen, tenoxicam, nifedipine, nimodipine, ursodeoxycholic acid, and various hydrophilic carriers such as polyethylene glycols (PEGs), ${ }^{[1-14]}$ polyvinylpyrrolidone (PVP) ${ }^{[15]}$ hydroxypropyl methylcellulose, gums, sugar, mannitol, urea, colloidal silicon dioxide, and lipids such as polyglycolized glycerides (Gelucire) ${ }^{[11,16,17]}$ have been investigated for improvement of dissolution characteristics and BA of poorly aqueous-soluble drugs. ${ }^{[18,19]}$ SDs are of two types: First generation and second generation. The first type of SD was described as formulation of eutectic mixtures which have been improved the rate of drug release and consequently the BA of poorly water-soluble drugs. ${ }^{[20]}$ Several SDs were described using poorly water-soluble drugs such as sulfathiazole $\mathrm{e}^{[8]}$ and chloramphenicol ${ }^{[20]}$ using urea as a high water-soluble carrier. Later, Levy ${ }^{[21]}$ and Kanig ${ }^{[22]}$ developed SD systems, containing mannitol as carrier, by preparing solid solutions through molecular dispersions instead of using eutectic mixtures. ${ }^{[2]}$ The improvements were attributed to faster carrier dissolution, releasing microcrystals or particles of drug. These SDs, which could be designated as first-generation SDs, were prepared using crystalline carriers. Crystalline carriers include urea and sugars, which were the first carriers to be employed in SDs. ${ }^{[22]}$ They have the disadvantage of forming crystalline SDs, which were more thermodynamically stable and did not release the drug as quickly as the amorphous ones. The concept of secondgeneration SD came in the late $60 \mathrm{~s}^{[10,23]}$ and it was observed that in SDs, the drug was maintained in the crystalline state. ${ }^{[24]}$ Various methods were employed for preparation of SDs, namely, solvent evaporation method, melt extrusion, spray drying, coprecipitation, fusion method, melting method, supercritical fluid methods, etc. The aim of the current study was to develop and characterize SD of spironolactone (SPL) as solubility and dissolution profile using conventional fusion method and compare it with marketed formulation.

\section{MATERIALS AND METHODS}

\section{Materials}

SPL was procured from Xiangu Pharmaceuticals, Zhejiang, China. PEG 4000, sodium hydroxide, sodium hydrogen phosphate, and hydrochloric acid $(\mathrm{HCl})$ were purchased from S.D. Fine Chemical, Pvt. Ltd., New Delhi, India and other reagents were of analytical grade.

\section{Preparation of physical mixture}

Physical mixtures (PMs) of SPL and PEG 4000 were made in different ratios of 1:1 w/w, 1:3 w/w, and 1:5 w/w by simple mixing process.

\section{Preparation of solid dispersion}

SD was prepared using the conventional fusion method. PEG 4000 was taken and heated to a molten state at $60^{\circ} \mathrm{C}$ and to this mass, the weighed amount of SPL was added with continuous stirring with a glass rod until dissolved. The different ratio of drug was added and finally SD was obtained in 1:5 w/w ratio of SPL to PEG 4000. Solidification was allowed to occur at room temperature. The product was stored in a Desiccators (Singhla scientific Industries, Haryana, India) for $24 \mathrm{~h}$ and then pulverized using a porcelain mortar and pestle (Dr. Scientific, Haryana, India). The pulverized powder was passed through 80-mesh sieve (Micro Teknic, Haryana, India) to get uniform particle size. ${ }^{[25]}$

\section{Solubility studies}

Phase and saturation solubility studies were conducted as per the method described by Higuchi et al ${ }^{[26]}$ The saturation solubility of drug, physical mixtures, and SD with PEG 4000 in distilled water and $0.1 \mathrm{~N} \mathrm{HCl}$ was determined by adding an excess of drug, physical mixture, and SD to $50 \mathrm{~mL}$ distilled water and $0.1 \mathrm{~N}$ $\mathrm{HCl}$ in a conical flask (Glassco, Delhi, India) and were shaken on rotary shaker (Remi Elektrotechnik Ltd, Mumbai, India) for $72 \mathrm{~h}$ at $37^{\circ} \mathrm{C} \pm 0.5^{\circ} \mathrm{C}$. The saturated solutions were filtered through a $0.45-\mu \mathrm{m}$ membrane filter (Membran Filter India Pvt, Ltd. Maharashtra, India), suitably diluted with water, $0.1 \mathrm{~N} \mathrm{HCl}$, and analyzed using Shimadzu UV-1601 (UV 1601 Shimadzu, Kyoto, Japan) UV-1601 spectrophotometer at 242 nm. ${ }^{[27]}$

\section{Differential scanning calorimetry study}

The degree of crystallinity of SPL and SD was determined using differential scanning calorimetry (DSC) study. The dried sample of SPL (about $1 \mathrm{mg}$ ) was loaded and sealed into DSC pan (Perkin Elmer Massachusetts, USA) with a DSC loading puncher (Perkin, Massachusetts, USA). The sample was scanned between $40^{\circ} \mathrm{C}$ and $400^{\circ} \mathrm{C}$ at a heating rate of $10^{\circ} \mathrm{C} / \mathrm{min}$, under nitrogen atmosphere. An empty pan was used as reference. An inert atmosphere was maintained by purging with nitrogen.

\section{Fourier transform infrared spectroscopy (FTIR)}

Infrared spectroscopy was conducted using Perkin Elmer (Massachusetts, USA) spectrophotometer, and the spectrum was recorded in the wavelength region of $6000-500 \mathrm{~cm}^{-1}$. The procedure consisted of dispersing a sample (drug alone or mixture drug and excipients) in $\mathrm{KBr}$ and compressing into discs by applying pressure. The pellet was placed in the light path, and the spectrum was obtained. It is used for confirming any interaction of SPL with other excipients. ${ }^{[28]}$

\section{In vitro dissolution studies}

Dissolution of pure SPL and SPL SD was performed using 8-station USP type-II apparatus (Veego Instruments, Mumbai, 
India) with paddle rotating at $100 \mathrm{rpm}$ at $37^{\circ} \mathrm{C} \pm 0.5^{\circ} \mathrm{C}$. The dissolution studies were conducted by filling the powder in a capsule and suspending the capsule in the basket in dissolution medium $(900 \mathrm{~mL}, 0.1 \mathrm{~N} \mathrm{HCl})$. Solid products, SDs as well as physical mixtures, each containing $25 \mathrm{mg}$ of equivalent drug were subjected to dissolution. The collected samples were filtered and replaced with fresh media at predetermined time points analyzed spectrophotometrically for the drug content at $242 \mathrm{~nm}$. Each sample was studied in triplicate $(n=3)$ for dissolution rate, and calculated mean values of cumulative drug release were used while plotting the release curves.

\section{Stability studies}

Stability studies were conducted as per the International Conference on Harmonization ( $\mathrm{ICH}$ ) guidelines. The samples were stored at $40^{\circ} \mathrm{C} \pm 0.5^{\circ} \mathrm{C} / 75 \pm 5 \%$ relative humidity $(\mathrm{RH})$ for 3 months, Thermolab Scientific Equipments Pvt. Ltd., Thane, Maharashtra, India. The samples were withdrawn and evaluated for the drug content and in vitro release at predetermined time intervals. The variations are analyzed and compared with the freshly prepared formulations. All samplings were taken in triplicates $(n=3)$.

\section{Statistical analysis}

All data are reported as mean \pm standard error of the mean (SEM) and the differences between the groups were tested using Student's $t$-test at the level of $P<0.05$. All groups were compared using analysis of variance (ANOVA) and the difference was greater at $P<0.05$ that was considered as significant.

\section{RESULTS AND DISCUSSION}

\section{Solubility studies}

The results of saturation solubility studies are given in Figure 1. The solubility of pure drug in water and in $0.1 \mathrm{~N} \mathrm{HCl}$ was found to be $23.54 \pm 1.75 \mu \mathrm{g} / \mathrm{mL}$ and $25.41 \pm 2.51 \mu \mathrm{g} / \mathrm{mL}$, respectively. The solubility of physical mixture prepared using PEG 4000 in the ratio $(1: 1,1: 3$, and $1: 5)$ was $27.94 \pm 2.11 \mu \mathrm{g} / \mathrm{mL}, 32.64 \pm$ $2.13 \mu \mathrm{g} / \mathrm{mL}$, and $37.74 \pm 1.63 \mu \mathrm{g} / \mathrm{mL}$, respectively, in water and

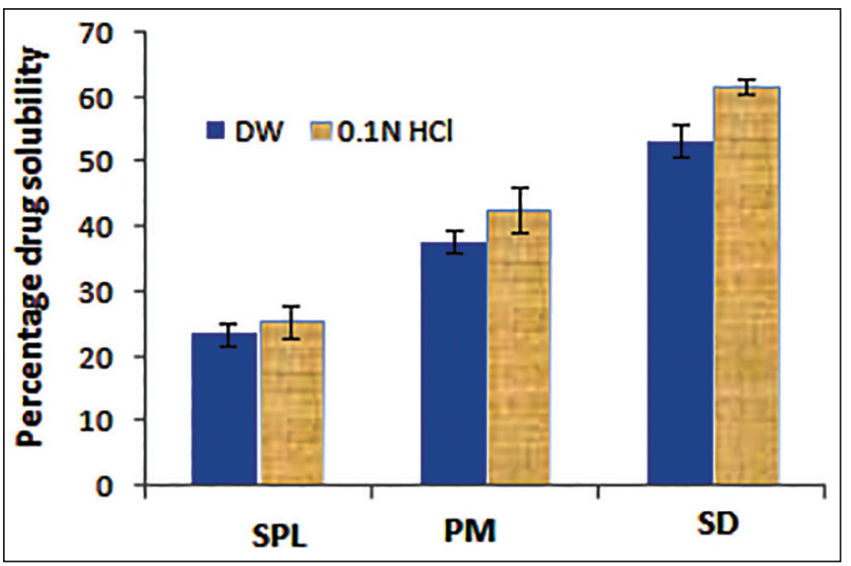

Figure 1: Results of saturation solubility with solid dispersion
$33.24 \pm 1.19 \mu \mathrm{g} / \mathrm{mL}, 36.64 \pm 4.32 \mu \mathrm{g} / \mathrm{mL}$, and $42.62 \pm 3.52 \mu \mathrm{g} / \mathrm{mL}$, respectively, in $\mathrm{HCl}$. The solubility of SD using PEG 4000 $(1: 1,1: 3$, and $1: 5)$ in water was found to be $40.33 \pm 2.18 \mu \mathrm{g} / \mathrm{mL}$, $46.24 \pm 1.16 \mu \mathrm{g} / \mathrm{mL}, 53.36 \pm 2.66 \mu \mathrm{g} / \mathrm{mL}$, respectively, and in $0.1 \mathrm{~N} \mathrm{HCl}$ was $42.74 \pm 3.21 \mu \mathrm{g} / \mathrm{mL}, 48.45 \pm 3.22 \mu \mathrm{g} / \mathrm{mL}$, and $61.73 \pm 1.26 \mu \mathrm{g} / \mathrm{mL}$ respectively. These results revealed that solubility was enhanced with SD using PEG. It is concurrent to the study conducted by other groups of scientists.

\section{Differential scanning calorimetry study}

DSC is commonly the thermal analysis method of choice because of its capability to provide detailed information about both the physical and energetic properties of a substance. ${ }^{[25]}$ It is the most preferred thermal technique for SD characterization, provides accurate information about MP, glass transition temperature $(\mathrm{Tg})$ as well as the energy, changes associated with the phase transitions including crystallization and fusion process. Absence of a drug melting peak in the DSC thermogram of SD indicated that the drug exists in an amorphous form or encapsulated form as seen in Figure 2. The previous studies showed that PEG was the most suitable excipient, which provides a single phase SD. DSC was used to know the molecular miscibility, recrystallization, and phase separation of SD after preparation and under accelerated stability conditions. In this study, another peak was observed with $\mathrm{SD}$ and showed molecular miscibility. The SD was physically stable if no recrystallization peaks were observed. ${ }^{[29]}$

\section{Fourier transform infrared (FTIR) study}

FTIR study was performed and the results were studied and analyzed. The pure SPL had shown a characteristic peak at infrared (IR) spectrum of SPL that revealed six bands at $1778 \mathrm{~cm}^{-1}, 1768 \mathrm{~cm}^{-1}, 1691 \mathrm{~cm}^{-1}, 1674 \mathrm{~cm}^{-1}, 1663 \mathrm{~cm}^{-1}$, and $1618 \mathrm{~cm}^{-1}$ in the double bond region. The $1691 \mathrm{~cm}^{-1}, 1674 \mathrm{~cm}^{-1}$, and $1618 \mathrm{~cm}^{-1}$ bands are assigned to the $\mathrm{C}=\mathrm{O}$ stretching of the

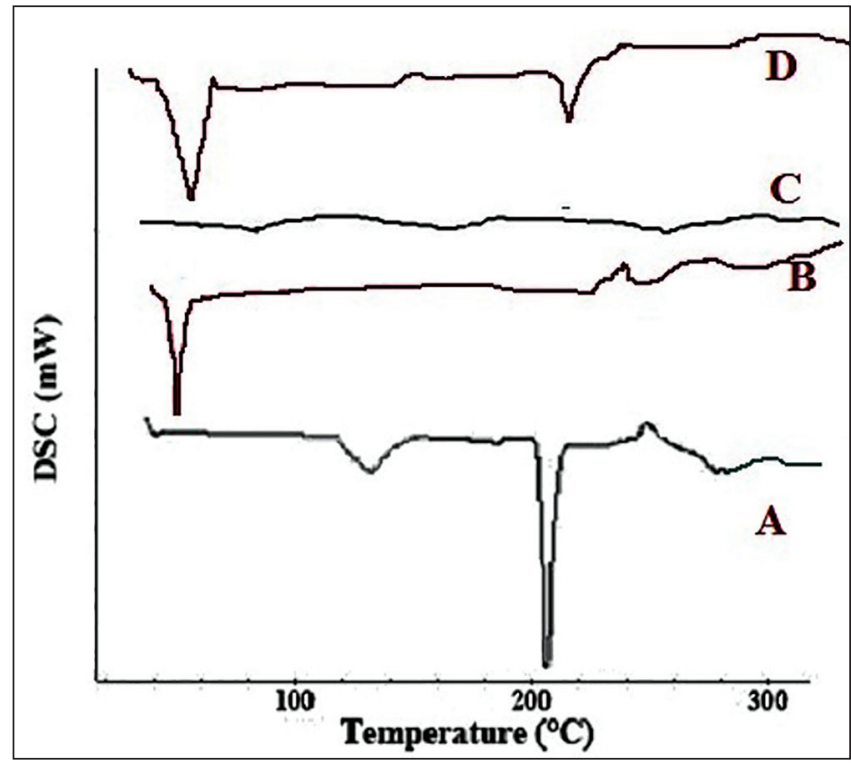

Figure 2: DSC thermograms of (a) SPL (b) PEG-4000 (c) SPL-solid dispersion (d) physical mixture 
thioacetyl group, $\mathrm{C}=\mathrm{O}$ stretching of the C6-ring, and $\mathrm{C}=\mathrm{C}$ stretching, respectively. The very strong IR band at $1768 \mathrm{~cm}^{-1}$ is attributed to the $\mathrm{C}=\mathrm{O}$ stretching of the lactone ring. The two weak bands at $1778 \mathrm{~cm}^{-1}$ and $1663 \mathrm{~cm}^{-1}$ are attributed to the overtone or combination bands as shown in Figure 3. However, SD with PEG 4000 was analyzed; the typical peaks were shifted, broadened, some had low intensity, and some were missed. It indicated the formation of SD with carriers because original peaks were vanished in the spectrum. It is a versatile technique to investigate the intermolecular interaction and drug-carrier compatibility because it can detect the physical and chemical reactions between the drug and carriers. H-bonding between the drug and carriers is an important characterization method to explain physical state and the stability of active pharmaceutical ingredient $(\mathrm{API})$ in SD. ${ }^{[30]}$

\section{Dissolution studies}

Dissolution studies are an important tool to characterize SD. The formulation with maximum homogeneity or intimate mixing and maximum amount of amorphous content of drug should show maximum dissolution. Pure API gave the least rate of dissolution; the maximum release rate was found in formulations containing polymer in the maximum ratio. PEG 4000 is a water-soluble carrier, which upon dissolution gives higher solubility of SD and ultimately more dissolution. The physical mixture gave more dissolution than pure API but lesser than SD due to the carrier effect in dissolution. In fusion technique, the molecular dispersion is formed due to the mixing caused by molecular mobility of drug and carrier molecules, which are greatest at the melting point of the two components of the dispersion. When a fused product is quench cooled molecular motion is arrested when mixing is at the utmost while gradual cooling at room temperature allows ample time for the drug and polymer molecules to segregate and settle as per their density considerations, giving a highly heterogeneous as well as recrystallized product. The dissolution release profiles of SPL for API, physical mixture, and SD were performed in $0.1 \mathrm{~N} \mathrm{HCl}(900 \mathrm{~mL})$ and the release profiles are shown as in Figure 4. The release rate was significantly increased when the SPL: PEG 4000 ratio was at 1:5. The maximum cumulative percentage release from pure drug, SPL marketed products (tablets), physical mixture, and SPL SD at 60 min was 27.25 $\pm 1.83 \%, 35.64 \pm 3.65 \%, 47.72 \pm 2.45 \%$, and $74.24 \pm 3.25 \%$, respectively, as shown in Figure 5 . The similar observations were also reported by Choudhary et al. ${ }^{[31]}$ The drug release from all the formulations followed first-order kinetics, as the plot observed in between log percentage drug remaining versus time was found to be linear with coefficient of correlation $\left(R^{2}\right.$ $=0.993$ ) as shown in Figure 6. To analyze the mechanism of drug release from these formulations, the data were followed Hixson-Crowell equation. The correlation coefficient ( $r$ ) values of the first-order release model are found to be (0.955-0.993) slightly higher when compared to the Hixson-Crowell's cube root model. Hence, the release of drug from the preparations followed predominantly first-order kinetics compared to Hixson-Crowell cube root law.

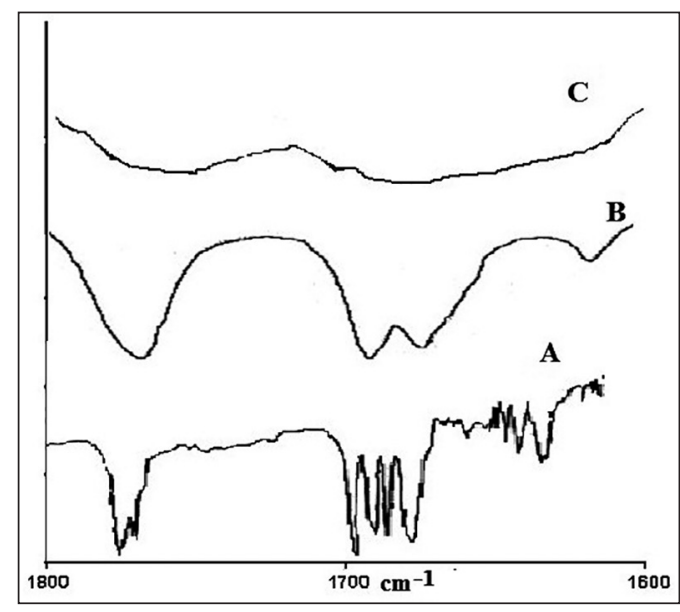

Figure 3: FT-IR spectra of (a) SPL (b) physical mixture (c) SPL solid dispersion

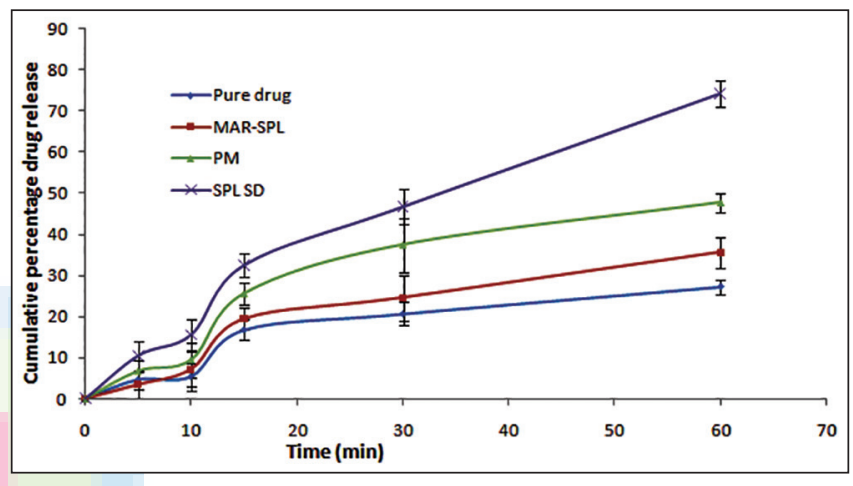

Figure 4: Dissolution profiles of SPL, physical mixture, marketed preparation, and SPL solid dispersion

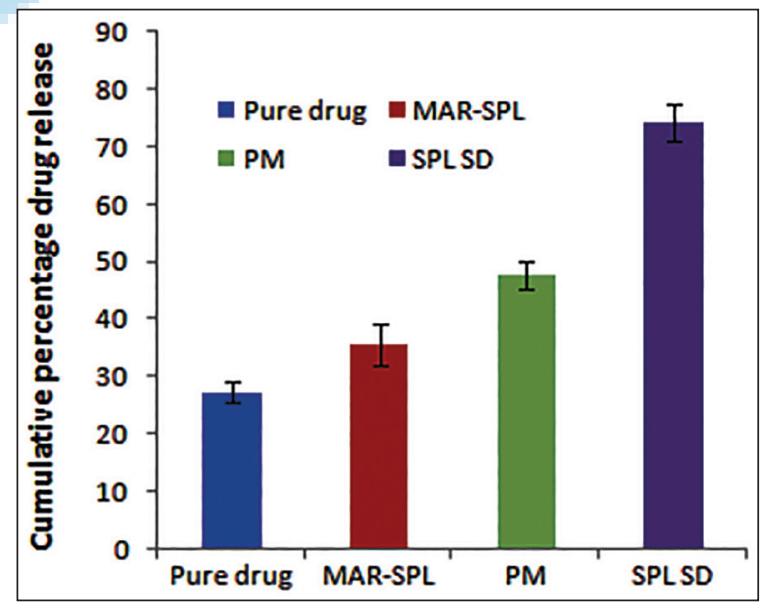

Figure 5: Maximum cumulative percentage release from SPL, SPL marketed product (tablets), physical mixture, and SPL solid dispersion at $60 \mathrm{~min}$

\section{Stability studies}

After removal of predetermined time interval, samples were analyzed for the content and in vitro release study and results were compared with the original data. No significant $(P<0.05)$ variations were found in the results. It is indicated that the prepared SD was stable under accelerated storage conditions. 

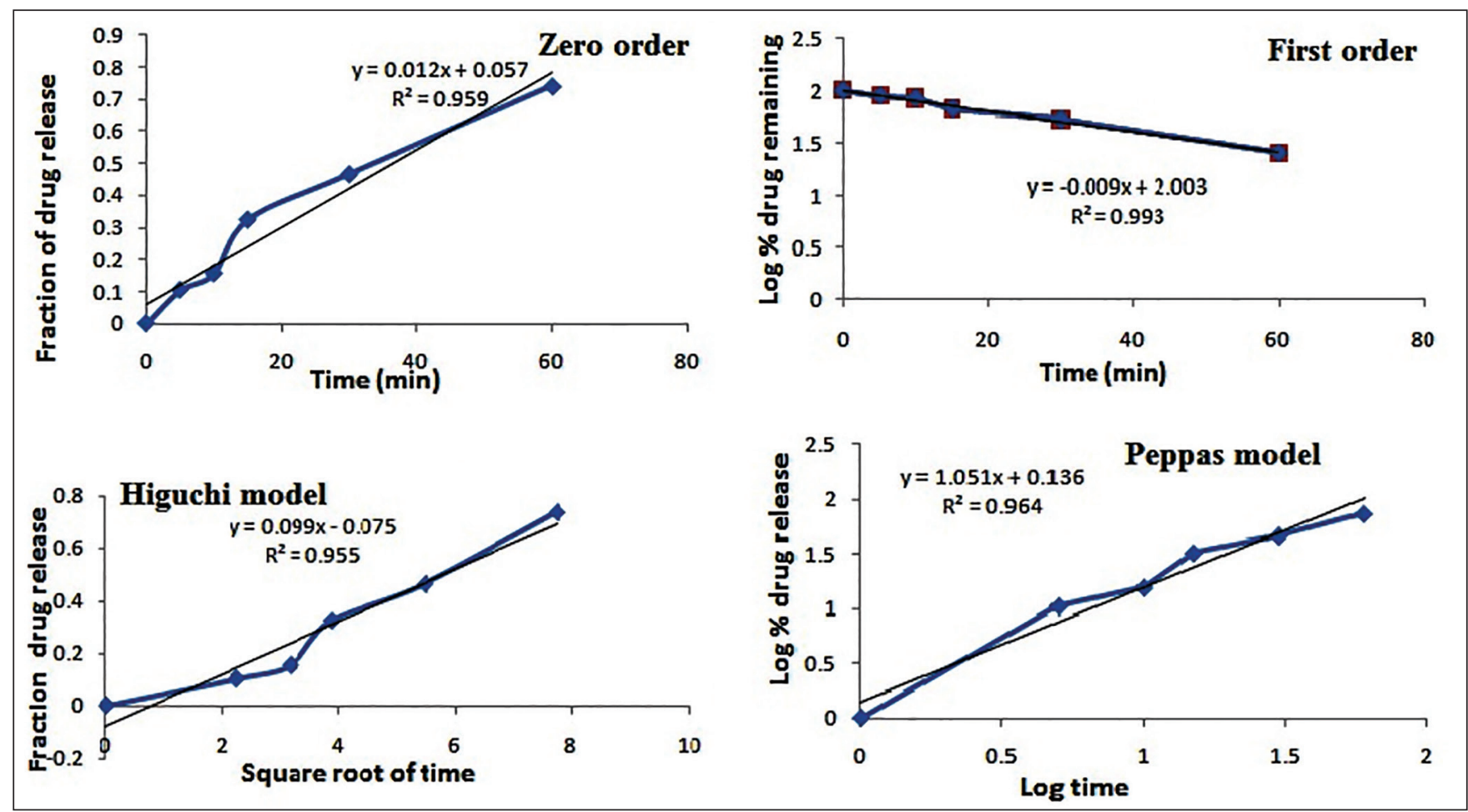

Figure 6: Various kinetics release profiles of solid dispersion

\section{CONCLUSION}

The SD of SPL was prepared using conventional fusion method. The solubility of SPL SD was found to be significantly increased $(P<0.05)$ as compared to SPL API and physical mixture of PEG 4000 and SPL. A marked cumulative percentage release was observed when compared with SPL marketed tablets and physical mixture. The prepared SD was characterized using DSC and XRD which showed absence of sharp drug peak in $\mathrm{SD}$ indicated that the drug physical property has been changed which is indicative of molecular interaction of PEG with SPL. The stability study revealed that prepared SD was stable because no significant loss $(<5 \%)$ of API was found during accelerated condition. Thus, the current study recommended that SD would be a good option for increasing solubility as well as bioavailability but the clinical study has been required before its clinical application in the upcoming days.

\section{Financial support and sponsorship}

Nil.

\section{Conflicts of interest}

There are no conflicts of interest.

\section{REFERENCES}

1. Jin T. Solid dosage forms for rapid dissolution of poorly soluble drugs. US Patent 20040001888.

2. Leuner C, Dressman J. Improving drug solubility for oral delivery using solid dispersion. Eur J Pharm Biopharm 2000;50:47-60.
3. Craig DQ. The mechanisms of drug release from solid dispersions in water-soluble polymers. Int J Pharm 2002;231:131-44.

4. Chauhan H, Hui-Gu C, Atef E. Correlating the behavior of polymers in solution as precipitation inhibitor to its amorphous stabilization ability in solid dispersions. J Pharm Sci 2013;102:1924-35.

5. Smikalla MM. Urbanetz NA. The influence of povidone K17 on the storage stability of solid dispersions of nimodipine and polyethylene glycol. Eur J Pharm Biopharm 2006;66:106-12.

6. Pignatello P, Ferro M, Puglisi G. Preparation of solid dispersions of nonsteroidal anti-inflammatory drugs with acrylic polymers and studies on mechanisms of drug-polymer interactions. AAPS PharmSci Tech 2002;3:E10.

7. Huang Y, Dai WG. Fundamental aspects of solid dispersion technology for poorly soluble drugs. Acta Pharm Sin B 2014:4:18-25.

8. Sekiguchi K, Obi N. Studies on absorptions of eutectic mixture. I. A comparison of the behavior of eutectic mixture of sulfathiazole and that of ordinary sulfathiazole in man. Chem Pharm Bull 1961;9:866-72.

9. Chiou WL, Riegelman S. Pharmaceutical applications of solid dispersion systems. J Pharm Sci 1971;60:1281-302.

10. Chiou WL, Riegelman S. Preparation and dissolution characteristics of several fast-release solid dispersions of griseofulvin. J Pharm Sci 1969;58:1505-10.

11. Joshi HN, Tejwani RW, Davidovich M, Sahasrabudhe VP, Jemal M, Bathala MS, et al. Bioavailability enhancement of a poorly water-soluble drug by solid dispersion in polyethylene glycol-polysorbate 80 mixture. Int J Pharm 2004;269:251-8.

12. Craig DQ, Newton JM. The dissolution of nortriptyline $\mathrm{HCl}$ from polyethylene glycol solid dispersions. Int $\mathrm{J}$ Pharm 1992,78:175-82.

13. Asyarie $\mathrm{S}$, Rachmawati $\mathrm{H}$. In vivo and in vitro evaluation of a solid dispersion system of gliclazide:PEG 6000. PDA J Pharm Sci Technol 2007;61:400-10. 
14. Patel RP, Patel MM. Physicochemical characterization and dissolution study of solid dispersions of Lovastatin with polyethylene glycol 4000 and polyvinylpyrrolidone K30. Pharm Dev Technol 2007;12:21-33.

15. Broman E, Khoo C, Taylor LS. A comparison of alternative polymer excipients and processing methods for making solid dispersions of a poorly water soluble drug. Int J Pharm 2001;222:139-51.

16. Kawakami K, Miyoshi K, Ida Y. Solubilization behavior of poorly soluble drugs with combined use of Gelucire 44/14 and cosolvent. J Pharm Sci 2004;93:1471-9.

17. Karata $\square$ A, Yüksel N, Baykara T. Improved solubility and dissolution rate of piroxicam using gelucire 44/14 and labrasol. Farmaco 2005;60:777-82.

18. Hirasawa N, Ishise S, Miyata H, Danjo K. Physicochemical characterization and drug release studies of nilvadipine solid dispersions using water-insoluble polymer as a carrier. Drug Dev Ind Pharm 2003;29:339-44.

19. Takafumi H, Fumie K, Ikuo F. Solid preparation comprising a solid dispersion that can be rapidly disintegrated and that allows a drug to be dissolved. US Patent Applicaton 20070248681.

20. Sekiguchi K, Obi N, Ueda Y. Studies on absorption of eutectic mixture. Il. Absorption of fused conglomerates of chloramphenicol and urea in rabbits. Chem Pharm Bull (Tokyo) 1964;12:134-44.

21. Levy $\mathrm{G}$. Effect of particle size on dissolution and gastrointestinal absorption rates of pharmaceuticals. Am J Pharm Sci Support Public Health 1963;35:78-92.

22. Kanig JL. Properties of fused mannitol in compressed tablets. J Pharm Sci 1964;53:188-92.
23. Simonelli AP, Mehta SC, Higuchi WI. Dissolution rates of high energy polyvinylpyrrolidone (PVP)-sulfathiazole coprecipitates. J Pharm Sci 1969;58:538-49.

24. Vippagunta SR, Wang Z, Hornung S, Krill SL. Factors affecting the formation of eutectic solid dispersions and their dissolution behavior. J Pharm Sci 2007;96:294-304

25. Zawar L, Bari S. Microwave induced solid dispersion as a novel technique for enhancing dissolution rate of repaglinide. Adv Pharmacol Pharm 2013;1:95-101.

26. Higuchi T, Connors KA. Phase solubility techniques. Adv Anal Chem Instrum 1965;4:117-212.

27. Narasaiah VL, Reddy BK, Kumar MR, Kumar AK, Raju Ch, Kumar AS, et al. Improved dissolution rate of atorvastatin calcium using solid dispersion with PEG-4000. J Chem Pharm Res 2010;2:304-11.

28. Miyazaki T, Aso Y, Yoshioka S, Kawanishi T. Differences in crystallization rate of nitrendipine enantiomers in amorphous solid dispersions with HPMC and HPMCP. Int J Pharm 2011;407:111-8

29. Vo CL, Park C, Lee BJ. Current trends and future perspectives of solid dispersions containing poorly water-soluble drugs. Eur J Pharm Biopharm 2013;85:799-813.

30. Miyazaki T, Yoshioka S, Aso Y, Kawanishi T. Crystallization rate of amorphous nifedipine analogues unrelated to the glass transition temperature. Int J Pharm 2007;336:191-5.

31. Choudhary A, Rana AC, Aggarwal G, Kumar V, Zakir F. Development and characterization of an atorvastatin solid dispersion formulation using skimmed milk for improved oral bioavailability. Acta Pharmceutic Sinica B 2012;2:421-8. 